\section{Comment on "Pressure Dependence of Fragile-to- Strong Transition and a Possible Second Critical Point in Supercooled Confined Water"}

In a recent Letter by Liu et al. [1], the authors present quasielastic neutron scattering (QENS) data on water confined in nanopores of MCM-41-S at different applied pressures. From their observed change of the temperature dependence of the relaxation time, from highly nonArrhenius at high temperatures to Arrhenius behavior in the deeply supercooled regime, they claim that they are observing a fragile-to-strong transition for the confined water. In this Comment, we do not question the possibility that some kind of liquid-liquid or fragile-to-strong transition occurs for supercooled bulk water; i.e., the temperature dependence of the glass-transition-related $(\alpha-)$ relaxation time changes from a pronounced non-Arrhenius behavior to a nearly Arrhenius temperature dependence in the temperature range $225-230 \mathrm{~K}$, as first suggested by Ito et al. [2]. However, we claim that Liu et al. [1] are not observing such a transition simply because they are not even observing the $\alpha$ relaxation in the deeply supercooled regime [3].

Since the fragility of a supercooled liquid (and, therefore, also a possible fragile-to-strong transition) refers to the temperature dependence of the glass-transition-related $(\alpha-)$ relaxation (or the directly related viscosity) of the supercooled liquid, it is possible to use this classification only when the $\alpha$ relaxation can be observed in the whole temperature range. However, if the Arrhenius fit to the measured relaxation times for temperatures below $225 \mathrm{~K}$ [see Fig. 2(a) in Ref. [1] for ambient pressure] is extrapolated to a relaxation time of approximately $100 \mathrm{~s}$ (which is commonly used to define the glass-transition temperature $T_{g}$ of a supercooled liquid), $T_{g} \approx 50 \mathrm{~K}$ is obtained. This is an unrealistically low glass-transition temperature [4], in strong conflict with all literature data on glass-transition and glass-transition-related dynamics of supercooled water. This implies that the measured relaxation times for $T<225 \mathrm{~K}$ cannot correspond to the $\alpha$ relaxation of the supercooled confined water. In further support for the absence of a glass-transition-related relaxation process in the deeply supercooled regime is that no calorimetric glass transition can be observed in MCM-41 of such small pore radii $(<10 \AA)$ [5]. Thus, we suggest that the apparent transition around $225 \mathrm{~K}$ is due to a confinement-induced vanishing of the $\alpha$ relaxation that occurs roughly in the same temperature range as the merged $\alpha-\beta$ relaxation of supercooled bulk water split into two separate processes with decreasing temperature. The relaxation times obtained in Ref. [1] for temperatures below $225 \mathrm{~K}$ should then correspond to a more local relaxation process of the supercooled confined water [3].

The interpretation that the apparent transition around $225 \mathrm{~K}$ is due to a vanishing of the $\alpha$ relaxation is further supported by the simultaneous transition in the $Q$ depen- dence at the same temperature (this is not shown in Ref. [1], but in a previous paper [6] by the same authors), since a local $\beta$-like relaxation is expected to exhibit a weaker $Q$ dependence. However, it should here be noted that the relaxation process observed in Ref. [1] at temperatures below $225 \mathrm{~K}$ is even faster (and has a lower activation energy) than the dielectrically observed $\beta$ relaxation of deeply supercooled water confined in MCM-41 [5,7] and other types of host materials [8]. This "universal" dielectric process of supercooled confined water has shown to reach a relaxation time of $100 \mathrm{~s}$ at about $130-140 \mathrm{~K}$ [8], rather than at $50 \mathrm{~K}$. Thus, the relaxation process observed in Ref. [1] using QENS, and more recently supported by a ${ }^{1} \mathrm{H}$ NMR study of the diffusion constant [9], at temperatures below $225 \mathrm{~K}$ cannot be due to the $\alpha$ relaxation (or even the same $\beta$ relaxation as dielectrically observed) of the deeply supercooled confined water. Instead, it has recently been shown [10] that this low temperature process observed by QENS and ${ }^{1} \mathrm{H}$ NMR extrapolates to a weak dielectric relaxation that arises from the motion of socalled Bjerrum-type defects [11]. These defects are present in both supercooled water and ice and are due to orientationally disordered water molecules that are hydrogen bonded to less than four other water molecules. Although such defects can migrate and give rise to proton diffusion, it is clear that this process cannot be classified as the $\alpha$ relaxation of deeply supercooled water, and consequently Liu et al. [1] are not observing any true fragile-to-strong transition.

Jan Swenson

Department of Applied Physics

Chalmers University of Technology

SE-412 96 Göteborg, Sweden

Received 23 September 2005; published 1 November 2006

DOI: 10.1103/PhysRevLett.97.189801

PACS numbers: 61.20.Lc, 61.12.Ex, 61.25.Em, 77.22.Gm

[1] L. Liu, S.-H. Chen, A. Faraone, C.-W. Yen, and C.-Y. Mou, Phys. Rev. Lett. 95, 117802 (2005).

[2] K. Ito, C. T. Moynihan, and C. A. Angell, Nature (London) 398, 492 (1999).

[3] J. Swenson, H. Jansson, and R. Bergman, Phys. Rev. Lett. 96, 247802 (2006).

[4] Y.Z. Yue and C.A. Angell, Nature (London) 427, 717 (2004).

[5] J. Hedström et al., J. Phys. IV (France) (to be published).

[6] A. Faraone, L. Liu, C.-Y. Mou, C.-W. Yen, and S.-H. Chen, J. Chem. Phys. 121, 10843 (2004).

[7] A. Spanoudaki, B. Albela, L. Bonneviot, and M. Peyrard, Eur. Phys. J. E 17, 21 (2005).

[8] S. Cerveny, G. A. Schwartz, R. Bergman, and J. Swenson, Phys. Rev. Lett. 93, 245702 (2004).

[9] F. Mallamace et al., J. Chem. Phys. 124, 161102 (2006).

[10] J. Swenson et al. (to be published).

[11] N. Bjerrum, Science 115, 385 (1952). 